\title{
ESTUDO CARPOLÓGICO DO CASTRO DE SÃO LOURENÇO (ESPOSENDE, NOROESTE DE PORTUGAL): PRIMEIROS RESULTADOS
}

\author{
João Pedro Tereso(1), Ana Almeida(2), Carlos Brochado de Almeida ${ }^{(3)}$, Pablo \\ Ramil ReGO(4), Filipe Costa VaZ ${ }^{(5)} \&$ Rubim Almeida DA Silva ${ }^{1}$
}

Resumo

\begin{abstract}
Durante a campanha de escavação que decorreu no Castro de São Lourenço, em 2008, foram recolhidas amostras sedimentares em três quadrículas do Sector D, junto à muralha, em níveis romanos do século IV. Um estudo carpológico permitiu identificar três cultivos: o trigo de grão nu (Triticum aestivum/durum), o milho-miúdo (Panicum miliaceum) e, provavelmente, o milho painço (Setaria cf. italica). O milho-miúdo é a espécie mais abundante e a única que surge em todas as amostras.

O milho e o trigo de grão nu apresentam diferentes características, o que potenciaria a optimização de diferentes áreas do território do povoado. Os três cultivos eram frequentes no noroeste peninsular durante a época romana.
\end{abstract}

Palavras-chave: São Lourenço; período romano; carpologia; agricultura.

\begin{abstract}
Fruits and seeds from Castro de São Lourenço (Esposende, northwest Portugal): first results. During the 2008 excavation in the roman settlement of Castro de São Lourenço, several soil samples were recovered from $4^{\text {th }}$ century AD levels in Sector D, next to the defensive wall. A carpologic study led to the identification of three crops: naked wheat (Triticum aestivum/durum), common millet (Panicum miliaceum) and probably foxtail millet (Setaria cf. italica). Common millet is the most abundant species and also the only one which was found in all samples.

Millet and naked wheat are crops with different traits which could have led to a most efficient use of this settlement's territory, allowing the exploitation of areas with distinct characteristics. All three species could be frequently found in northwest Iberia during the Roman period.
\end{abstract}

Key-words: São Lourenço; roman period; carpology; agriculture.

\section{INTRODUÇ̃̃o}

Na campanha de escavação arqueológica realizada no Castro de São Lourenço no ano de 2008 foram recolhidas amostras sedimentares com vista à realização de um estudo de Arqueobotânica. Pretendia-se juntar informação relevante acerca das práticas agrícolas e também da paisagem envolvente do povoado.

O estudo cingir-se-á, no entanto, aos resultados do estudo carpológico. Este tinha como objectivo abordar questões de ordem paleoeconómica, nomeadamente obter informações relevantes acerca da agri- cultura praticada pelas comunidades humanas que habitaram São Lourenço em época romana.

Este estudo enquadra-se no projecto de doutoramento do primeiro autor, intitulado "Interacção entre flutuações climáticas, evolução da paisagem e dinâmicas de exploração antrópica do território nos últimos três milénios: o caso do NW peninsular". Este projecto conta com o apoio da FCT (bolsa de investigação no âmbito do QREN - POPH - Tipologia 4.1 - Formação Avançada, comparticipado pelo Fundo Social Europeu e por fundos nacionais do MCTES).

O povoado de S. Lourenço localiza-se no monte de S. Lourenço, abrangendo as freguesias de Mari-

(1) Universidade do Porto, Faculdade de Ciências, Departamento de Biologia, Edifício FC4, Sala 1.29, Rua do Campo Alegre, S/N, 4169-007 Porto, Portugal: CIBIO - Research Center In Biodiversity and Genetic Resources. jptereso@gmail.com

(2) Câmara Municipal de Esposende, Divisão de Cultura e Turismo, Serviço de Património Cultural, Praça do Município, 4740-223 Esposende, Portugal. arqueologia@cm-esposende.pt | ana.almeida@cm-esposende.pt

(3) Universidade do Porto, Faculdade de Letras, Departamento de Ciências e Técnicas do Património, Via Panorâmica, s/n, 4150-564 Porto, Portugal. calmeida@letras.up.pt

(4) Laboratorio de Botánica \& Bioxeografía. Instituto de Biodiversidade Agraria e Desenvolvemento Rural (IBADER). Universidade de Santiago de Compostela. ramil.rego@usc.es

(5) Faculdade de Letras, Universidade do Porto. filipe.mcvaz@gmail.com 
nhas e de Vila Chã, concelho de Esposende, distrito de Braga. Sobranceiro à plataforma litoral e ao Oceano Atlântico, constitui uma das elevações da arriba fóssil, posicionando-se a uma altitude de $203 \mathrm{~m}$ (Figs. 1 e 2).

Como tantos outros povoados do Entre-Douro-e-Minho, o castro de S. Lourenço tem as suas raízes no Bronze Final, mais especificamente no período balizado entre os séculos VII e VI a.C. O espaço temporal melhor conhecido deste habitat é o que medeia os séculos II a.C. e I d. C.

$\mathrm{O}$ sistema defensivo, composto por três ordens de muralhas e fossos no quadrante Nor-Noroeste, terá sido consolidado na primeira centúria da Era actual. A defesa integra ainda um torreão de vigilância, situado num dos cabeços a Noroeste do povoado, providenciando uma visualização profícua para a vertente norte da orla litoral.

Tudo indica que o castro de S. Lourenço se adaptou às novas realidades impostas pela administração romana. Após o séc. I d.C. terá sido convertido em vicus, uma nova realidade que se caracteriza por uma estrutura de carácter urbano, com funções de comércio e mesmo de pequena administração. Paralelamente prestaria serviços a todos os que habitavam o povoado, bem como os que optaram pelo abandono do castro para se instalarem, dispersamente, pela mancha agrícola adjacente.

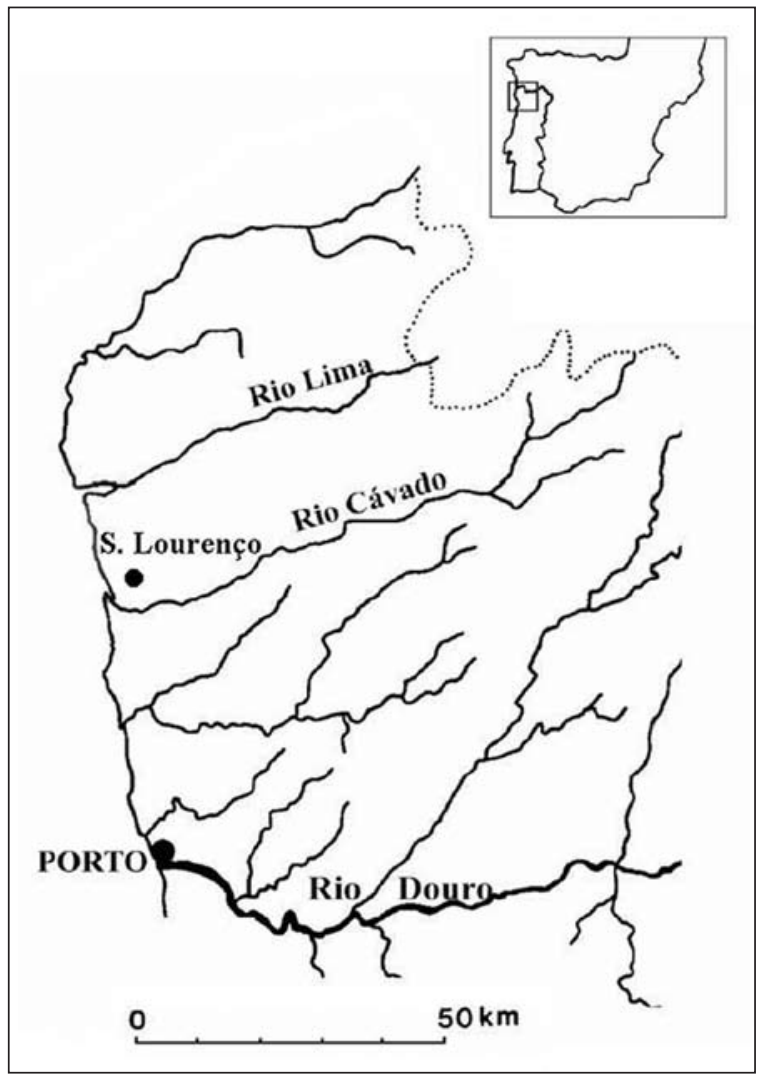

Fig. 1. Localização de São Lourenço na Península Ibérica. Fig. 1. Position of São Lourenço in the Iberian Peninsula.

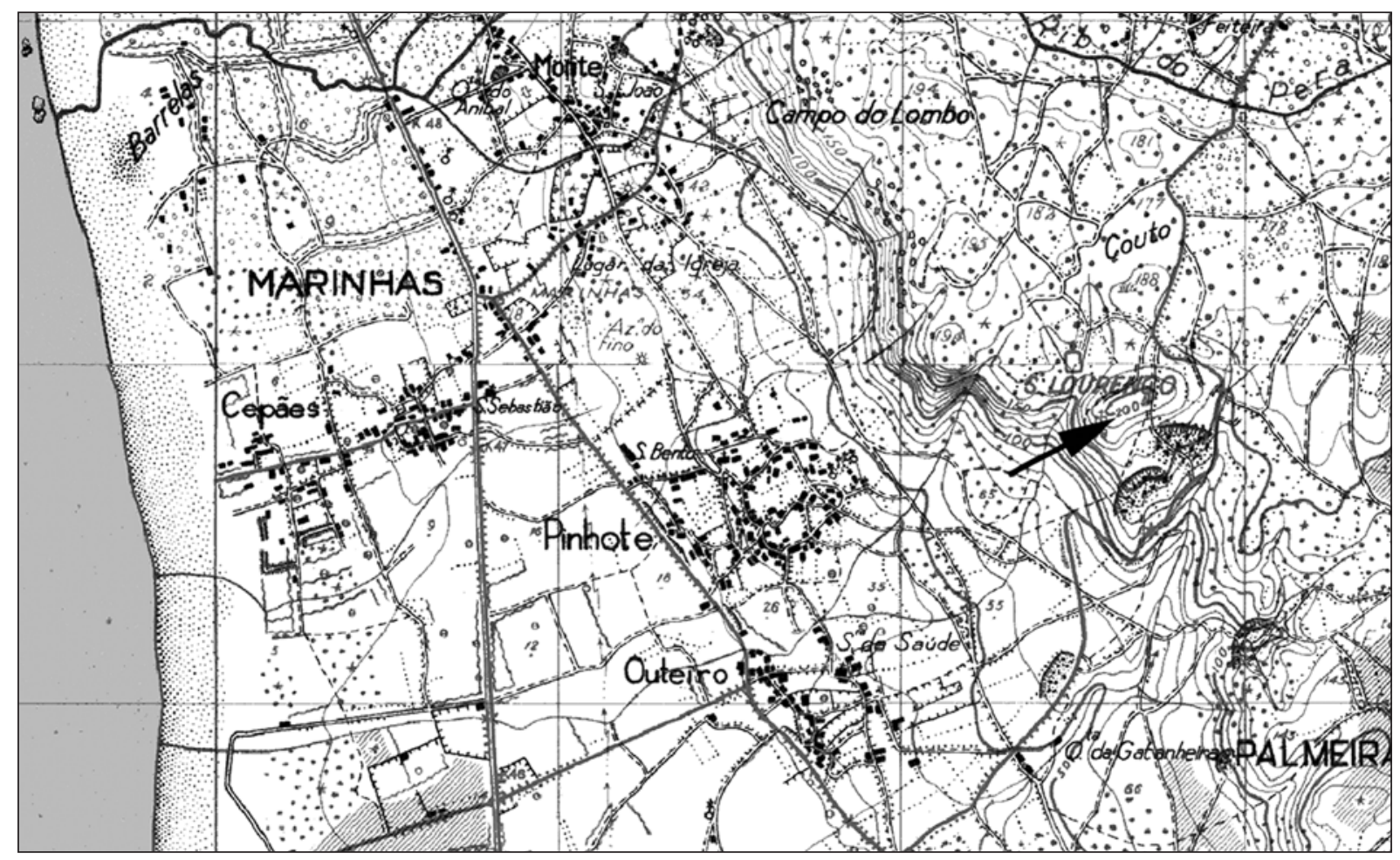

Fig. 2. Pormenor de localização de São Lourenço (CMP de Portugal, folhas 54 e 68), esc. 1:25 000.

Fig. 2. Detail of the position of São Lourenço (CMP of Portugal $54^{\text {th }}$ and $68^{\text {th }}$ ), scale 1:25 000 . 
A sua ocupação perdurou até ao início da Alta Idade Média (séc. V), momento em que a população residente terá optado definitivamente pela dispersão habitacional.

Após um período de abandono, o monte de S. Lourenço volta a ser ocupado de forma pontual entre os séculos XII e XIV. É neste período que é erguido um pequeno castelo, integrando a rede de encastelamento da Reconquista (ALMEIDA 2008).

\section{Descrição do sector D}

Situado no espaço Nordeste, adjacente ao aglomerado granítico onde está implantada a capela de $\mathrm{S}$. Lourenço, o sector D deriva a sua designação ao seu carácter Defensivo.

Neste sector foi identificada uma muralha - designada de M1 - parcialmente posta a descoberto entre 2001 e 2003. Com as faces externa e interna bem definidas por uma fiada de pedras de grande porte, unidas com terra, apresenta uma largura média de $4,80 \mathrm{~m}$ e uma altura variável entre os $0,80 \mathrm{~m} \mathrm{e}$ os $2,80 \mathrm{~m}$.

No espaço interior contíguo à muralha foi identificado um conjunto de edifícios, cujas funções estão presentemente em estudo. O último nível de ocupação desta área, que corresponde à maioria das estruturas pétreas de planta quadrangular e rectangular, remontará ao Baixo-Império. A cronologia foi aferida pelos numismas exumados, ainda inéditos. Estes são atribuíveis ao Imperador Constantino Magno. Tendo governado o Império Romano entre 306 e 337, a presença de moedas produzidas sob o seu cunho garantem a ocupação desta zona do povoado no séc. IV e mesmo na centúria seguinte, caso a circulação destes numismas tenha ultrapassado a vigência do reinado de Constantino.

\section{Materiais e Métodos}

Foram recolhidas amostras sedimentares com vista à detecção de macrorrestos vegetais - carvões, sementes e frutos. Ainda assim, até ao presente momento só os vestígios carpológicos foram alvo de um estudo sistemático que aqui se apresenta. As amostras sedimentares serão referenciadas pela sua localização na área de escavação (quadrícula) e enquadramento estratigráfico (unidade estratigráfica - u.e.).

As amostras sedimentares foram recolhidas em três quadrículas do sector $\mathrm{D}$, junto à muralha (ver Fig. 3 e Tabela 1):

- Quadrícula D8: foram recolhidas duas amostras na u.e. 3, um depósito cinzento-escuro, pouco compacto, com visíveis macrorrestos vegetais e sem espólio artefactual. Cobrindo directamente um nível de circulação composto por saibro, esta u.e. 3 foi interpretada como um nível de ocupação/abandono. Tendo-se recolhido duas amostras da mesma realidade arqueológica, estas foram designadas de Am. 1 e Am. 2.

A Am.1 foi recolhida durante uma decapagem em toda a extensão da u.e., enquanto a Am. 2 foi recolhida numa zona de concentração de macrorrestos.

- Quadrícula K7: das cinco amostras recolhidas nesta quadrícula só uma, da u.e. 6, continha material carpológico. Esta foi recolhida junto a um piso, na área sudeste da quadrícula. Trata-se de um depósito castanho-claro, medianamente compacto, por vezes compacto, com visíveis macrorrestos vegetais, cerâmicas de cariz doméstico e escassa cerâmica de construção.

- Quadrícula L7: também aqui foram recolhidas cinco amostras, tendo-se detectado vestígios carpológicos em duas delas, nomeadamente das u.e. 7 e 12. A u.e. 7 corresponde a um depósito saibrento castanho-claro, medianamente compacto, interpretado como nível de circulação. A u.e. 12 é um depósito cinzento, pouco compacto e com visíveis macrorrestos vegetais, sendo interpretado como nível de ocupação. A amostra da u.e. 12 foi recolhida junto a uma lareira (L1) composta por barro cozido.

Como é perceptível pela descrição efectuada, somente uma das amostras se encontrava associada a uma estrutura de combustão. De facto, as restantes amostras estudadas correspondem a recolhas pontuais em contextos sedimentares dispersos, o que condiciona a interpretação dos resultados obtidos.

No que respeita à inserção cronológica dos contextos em questão, a sua associação a numismas atribuíveis ao imperador Constantino Magno (vide supra) sugerem uma cronologia do século IV ou V.

Não havendo possibilidade de processar as amostras sedimentares junto ao sítio arqueológico optou-se pela recolha de amostras de pequenas dimensões, entre 2 e 15 litros. A pequena dimensão das amostras condiciona a interpretação das mesmas, mas permite testar o potencial deste tipo de estudo para futuras campanhas no Castro de São Lourenço.

As amostras sedimentares foram posteriormente flutuadas manualmente nos laboratórios do Departamento de Biologia da Faculdade de Ciências da Universidade do Porto tendo-se utilizado para o efeito colunas de crivos com malhas de $0,25 \mathrm{~mm}, 0,5 \mathrm{~mm}$, $1 \mathrm{~mm}$ e $2 \mathrm{~mm}$. 


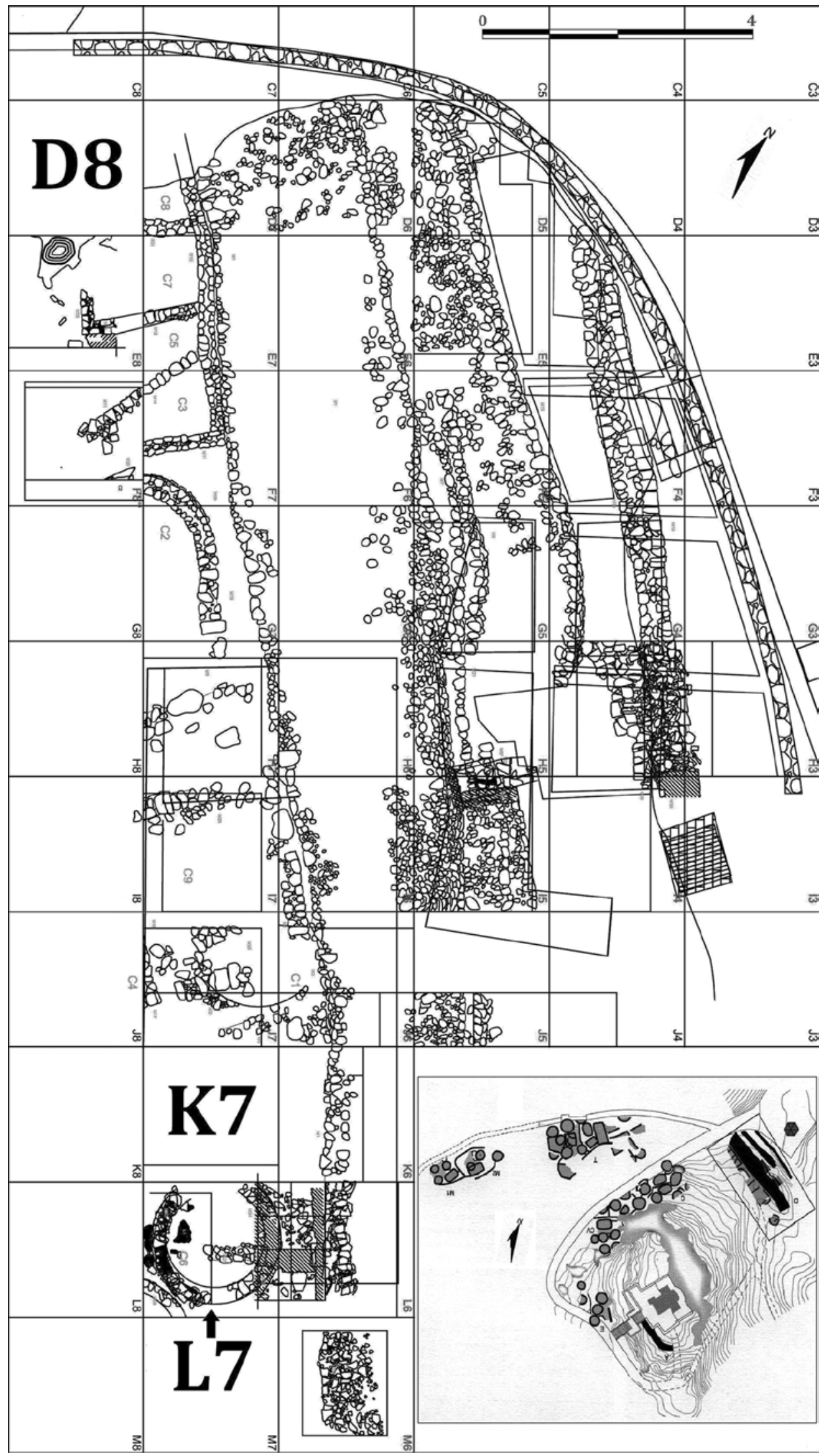

Fig. 3. Planta do Sector D.

Fig. 3. General view over Sector D. 
Tabela 1. Material carpológico recolhido.

Table 1. Carpological material.

\begin{tabular}{|c|c|c|c|c|c|c|}
\hline & \multicolumn{2}{|c|}{ D8 - u.e. 3} & \multirow{2}{*}{ K7 - u.e. 6} & \multirow{2}{*}{ L7 - u.e. 7} & \multirow{2}{*}{ L7 - u.e. 12} & \multirow{2}{*}{ TOTAL } \\
\hline & Am. 1 & Am.2 & & & & \\
\hline \multicolumn{7}{|l|}{ Cultivos } \\
\hline Cereal & & & 1 & & & 1 \\
\hline Panicum miliaceum & 4 & 2 & 2 & 2 & 13 & 23 \\
\hline Panicum/Setaria & & & 1 & & 12 & 11 \\
\hline Setaria cf. italica & & & 2 & & 24 & 26 \\
\hline Triticum sp. & & 1 & 1 & & & 2 \\
\hline Triticum aestivum/durum (grão) & 2 & & & & & 2 \\
\hline Triticum aestivum/durum (ráquis) & 4 & & & & & 4 \\
\hline \multicolumn{7}{|l|}{ Plantas silvestres } \\
\hline Asteraceae & 1 & & & & & 1 \\
\hline Chenopodiaceae & 1 & & & & & 1 \\
\hline Gramineae & 1 & & & & 2 & 3 \\
\hline Indeterminado & 11 & 4 & & 2 & 11 & 28 \\
\hline TOTAL & 24 & 7 & 7 & 4 & 60 & 102 \\
\hline
\end{tabular}

O produto da flutuação foi seco em estufa, procedendo-se depois à observação e triagem à lupa binocular com vista à recolha de frutos e sementes.

A identificação dos frutos e sementes foi efectuada com recurso à colecção de referência em montagem no CIBIO/FCUP, assim como a atlas anatómicos (e.g. JACOMET 2006) e diversas obras da especialidade (e.g. BuXó 1997).

As cariopses de trigo de grão nu bem como os fragmentos de ráquis de pequena dimensão, impossíveis de descriminar ao nível da espécie, surgem sob a designação Triticum aestivum/durum (BUXÓ 1997). Esta designação pretende incluir diversas espécies, nomeadamente, Triticum aestivum subsp. vulgare (Vill) Mackey, Triticum turgidum conv. durum (Desf.) Mackey e Triticum turgidum conv. turgidum (L.) Mackey.

\section{Resultados}

A generalidade das amostras estudadas era pobre em material carpológico (ver tabela 1). Os frutos e sementes detectados encontravam-se carbonizados, mas em considerável mau estado de preservação, o que justifica a quantidade significativa de elementos que não foi possível identificar ( $27 \%$ do conjunto carpológico).

Como é possível perceber pela leitura da tabela 1 , demonstra-se a presença de pelo menos três cultivos distintos, Panicum miliaceum (milho-miúdo), Setaria cf. italica (milho painço) e Triticum spp. (trigo).

A distinção das duas espécies de milho nem sem- pre foi fácil. Optámos pelos critérios morfológicos definidos por R. Buxó (1997) e S. Jacomet (2006). Os grãos de Panicum miliaceum apresentam uma forma elipsoidal a arredondada, apresentando o hilo redondo e o escutelo largo, tendencialmente oval e não ultrapassando a metade do comprimento do grão (Figura 4, esquerda). Por sua vez, os grãos de género Setaria apresentam maior dificuldade ao nível da identificação específica. Os critérios que permitem distinguir a espécie cultivada (Setaria italica) de outras plantas do mesmo género (vide BuXó 1997) podem ser difíceis de identificar em conjuntos numericamente pouco expressivos e em mau estado de preservação. No caso do conjunto de macrorrestos de São Lourenço, os grãos de Setaria apresentam dimensões ligeiramente menores que os de Panicum

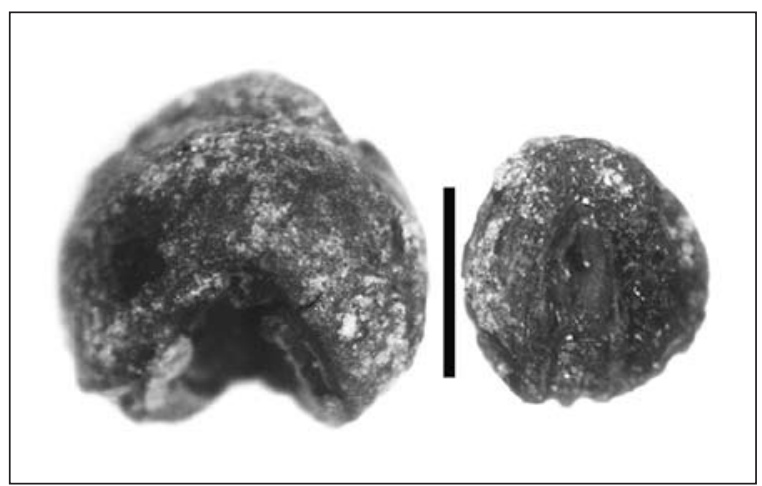

Fig. 4. Grão de Panicum miliaceum (esquerda) e Setaria cf. italica (direita).

Fig. 4. Grains from Panicum miliaceum (left) and Setaria cf. italica (right). 
miliaceum, têm um hilo mais comprido do que largo e um escutelo mais estreito e comprido, ultrapassando frequentemente a metade do comprimento do grão. Tratam-se muito provavelmente de grãos de Setaria italica, embora, pelos motivos expostos, não se possa excluir por completo a hipótese de se tratar de outra espécie do mesmo género.

No que respeita aos vestígios de trigo, não foi possível determinar qual a sua espécie. No entanto, é claro pela morfologia dos grãos e dos fragmentos de ráquis que estamos perante trigos de grão nu. Os grãos identificados como Triticum aestivum/durum (Fig. 5) apresentam forma oval, sendo ligeiramente convexos na sua face ventral vista de perfil, surgindo com o seu característico aspecto inchado (BUXÓ 1997; JACOMET 2006).

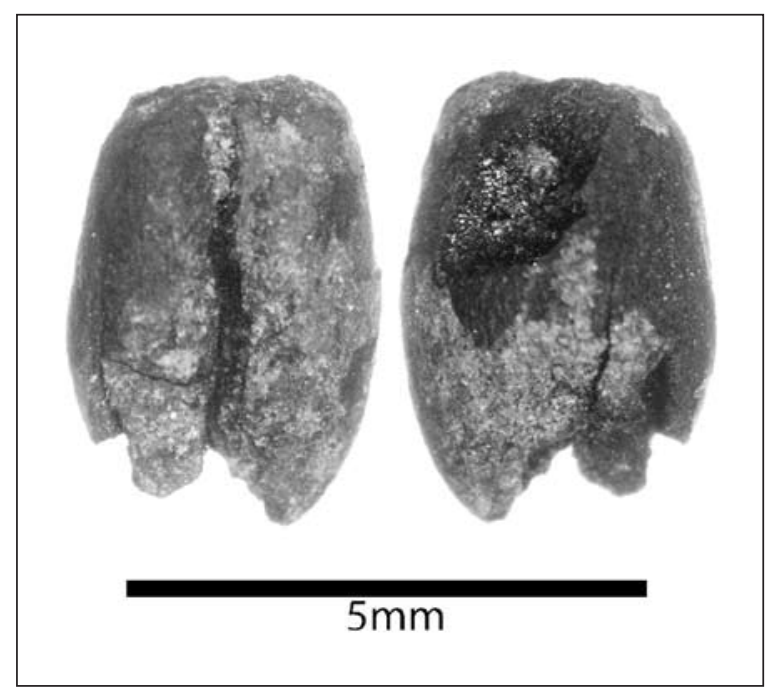

Fig. 5. Grão de Triticum aestivum/durum.

Fig. 5. Triticum aestivum/durum (grain).

É sabido que a análise de fragmentos de ráquis constitui a melhor forma de descriminar entre trigos nus tetraploides e hexaploides (HILLMAN et al. 1996). No entanto, os quatro fragmentos de nós que foram recolhidos numa das amostras da quadrícula D8 apresentam dimensões demasiado pequenas, faltando os elementos essenciais para uma segura discriminação dos tipos morfológicos.

Por fim, as sementes de espécies silvestres não potenciam a obtenção de informação relevante para este estudo. De facto, como é apontado na tabela 1 , não foi possível fazer uma identificação de qualquer um dos cinco carporrestos ao nível da espécie. Ainda que diversas espécies das famílias em questão sejam sinantrópicas e estejam relacionadas com práticas de cultivo, a adscrição ao nível da família não permite confirmar esta interpretação.

\section{Discussão e ConClusões}

O Panicum miliaceum é a espécie mais abundante nas amostras estudadas, sendo também a única que surge na totalidade das amostras, atestando a sua importância no período em questão. Os milhos foram cultivos de significativa importância no noroeste peninsular, onde surgem pela primeira vez durante a Idade do Bronze (BETTENCOURT 1999, BETTENCOURT et al. 2007). As duas espécies de milho - Panicum miliaceum e Setaria italica - surgem juntas nos povoados romanos da Terronha de Pinhovelo (Macedo de Cavaleiros) (Tereso 2007, 2008, 2009) e de Monte Mozinho (Penafiel) (TERESO et al. 2010).

Os milhos (Panicum/Setaria) são cultivos de Primavera, ou seja, são semeados na Primavera e colhidos no final do Verão. São espécies com ciclo vegetativo curto que se adaptam a diversos tipos de solos e condições climáticas, ainda que exijam alguma humidade no solo nas etapas iniciais do seu desenvolvimento (CHOPRA \& PRAKASH 2003).

O Panicum miliaceum e a Setaria italica podem ser cultivados e até confeccionados juntos, o que pode justificar o seu surgimento em conjunto (MARINVAL 1992). Podem ser utilizados para o fabrico de papas e até de pão, tal como é feito hoje em dia. Tal utilização surge mencionada por Plínio (apud RENFREW 1973).

No Castro de São Lourenço, a amostra onde os milhos surgem em maior número é a única que está associada a uma estrutura de combustão, na quadrícula L7, u.e. 12. Trata-se, na verdade, do contexto com maior número de vestígios carpológicos e a ausência de outros cereais afere a sua homogeneidade. Acrescente-se que a escassez de sementes nas restantes amostras parece confirmar o seu carácter ocasional, em nítido contraste com a amostra da u.e. 12 da quadrícula L7.

O trigo nu (Triticum aestivum/durum) surgiu na região durante o Neolítico, assumindo desde cedo, a par da cevada, um papel determinante nos sistemas agrícolas das comunidades humanas do noroeste (RAmil Rego \& Aira Rodriguez 1993). Durante a Idade do Ferro e época romana a preponderância do trigo tornou-se mais evidente (RAMIL REGO et al. 1996).

No Castro de São Lourenço os vestígios de trigo são escassos: 4 grãos e 4 fragmentos de ráquis que, ainda assim, não foi possível identificar além do tipo morfológico genérico de Triticum aestivum/durum. A existência de sub-produtos do processamento de trigo poderá indicar que as actividades de limpeza do grão poderão ter decorrido no interior do povoado.

Os trigos de grão nu preferem zonas de clima temperado sendo o seu desenvolvimento limitado 
pelas geadas. Necessitam de precipitações na ordem dos $500 \mathrm{~mm}$, preferencialmente no período primaveril, podendo, por outro lado, ser negativamente afectados pelo excesso de calor e falta de água durante a fase de desenvolvimento das espigas (CHOPRA \& PRAKASH 2003). Não obstante, existem variedades de trigo adaptadas ao cultivo primaveril.

A relativa pobreza carpológica dos contextos estudados não permite abordagens mais arrojadas ao sistema agrícola das comunidades do Castro de São Lourenço no século IV, nem à compreensão dos contextos arqueológicos abordados. De qualquer forma, é provável que as referidas comunidades tenham consumido e, eventualmente, cultivado duas espécies de milho (Panicum miliaceum e Setaria italica) e trigo de grão nu. Pelas características intrínsecas destas espécies, sabemos que certamente potenciariam a exploração agrícola de diferentes áreas do território do povoado.

O presente estudo cumpriu o objectivo de demonstrar o potencial deste tipo de análise no sítio arqueológico em questão, ficando clara, no entanto, a necessidade de dar continuidade a este tipo de abordagem em trabalhos futuros. Futuros trabalhos deverão ser conduzidos de forma a aumentar o esforço de amostragem no sentido de incluir um maior número e variedade de contextos e aumentar de forma significativa o volume de sedimentos a analisar.

\section{BIBLIOGRAFIA}

Almeida, A \& Almeida, C. 2008. Castro de S. Lourenço-Esposende: Câmara Municipal de Esposende.

BetTENCOURT, A.M.S. 1999 A paisagem e o Homem na bacia do Cávado durante o II e o I milénio AC. Braga: Dissertação de doutoramento apresentada à Universidade do Minho.

Bettencourt, A.M.S.; Dinis, A.; Figueiral, I.; Rodrigues, A.; Cruz, C.; Silva, I.; Azevedo, M. \& Barbosa, R. 2007. A ocupação do território e a exploração de recursos durante a Pré-história Recente do Noroeste de Portugal. In S.O. Jorge, A.M.S. Bettencourt \& I. Figueiral (eds.) A Concepção das paisagens e dos espaços na Arqueologia da Península Ibérica. Actas do IV Congresso de Arqueologia Peninsular. Promontoria Monográfica 08. Faro: 149-164.
BuXÓ, R. 1997. Arqueologia de las plantas. Barcelona: Crítica.

CARTA Militar de Portugal, Esc. 1:25.000. Serviços Cartográficos do Exército, Lisboa Folha: 68.

Chopra, V.L. \& Prakash, S. 2003. Evolution and Adaptation of Crops. Vol 1. Cereals. USA: Science Publishers Inc.

Hillman, G.; Mason, S.; De Moulins, D. \& Nesbitt, M. 1996. Identification of archaeological remains of wheat: the 1992 London workshop. Circaea 12 (2): 195-209.

JACOMET, S. 2006. Identification of cereal remains from archaeological sites (2 ${ }^{\text {nd }}$ edition).

MARINVAL, P. 1992. Archaeobotanical data on millets (Panicum miliaceum and Setaria italica) in France. Review of Palaeobotany and Palynology 73: 259-270.

QueIRogA, F. 1992. War and Castros. New approaches to the northwestern Portuguese Iron Age. PhD.

Ramil Rego, P.; Dopazo Martínez, A. \& Fernández RodríGUEZ, C. 1996. Cambios en las estrategias de explotación de los recursos vegetales en el Norte de la Península Ibérica. In P. Ramil Rego \& C. Fernández Rodríguez (coords.) Arqueometría y paleoecología del Norte de la Península Ibérica. Cambios naturales y perturbaciones antrópicas. Férvedes 3: $9-31$.

RAMIL-REgO, P. \& AIRA RodrigueZ, M. 1993. A palaeocarpological study of Neolithic and Bronze Age levels of the Buraco da Pala rock-shelter (Bragança, Portugal). Vegetation History and Archaeobotany 2: 163-172.

RENFrEW, J. 1973. Palaeoethnobotany. The prehistoric food plants of the Near East and Europe. New York: Columbia University Press.

TERESO, J. 2007. Economia agrícola das comunidades romanas do NW peninsular: dados carpológicos da Terronha de Pinhovelo (Bragança, Portugal). Recursos Rurais 1 (3): 13-21.

TERESO, J. 2008. Estudo arqueobotânico das estruturas romanas da Terronha de Pinhovelo (NE Portugal). Actas de 1 Congreso Internacional de Arqueoloxía de Vilalba. Férvedes 5: 69-78.

TERESO, J. 2009. Plant macrofossils from the roman settlement of Terronha de Pinhovelo (NW Iberia). Vegetation History and Archaeobotany 18: 489-501.

Tereso, J.; Carvalho, T.; Almeida Da Silva, R. \& Ramil REgo, P. 2010. Cultivos e armazenagem em Monte Mozinho: dados preliminares. In A.M.S. Bettencourt, M.I.C. Alves \& S. Monteiro-Rodrigues (eds.) Variações paleoambientais e evolução antrópica no Quaternário do Ocidente Peninsular / Palaeoenvironmental changes and anthropisation in the Quaternary of Western Iberia. Braga: Associação Portuguesa para o Estudo do Quaternário - APEQ e Centro de Investigação Transdisciplinar. Cultura, Espaço e Memória - CITCEM: 149-158. 